УДК 656.025: 519.17

08.00.00 Экономические науки

МОДЕЛИРОВАНИЕ

КРУПНОМАСШТАБНОЙ ТРАНСПОРТНОЙ СЕТИ ПРЕДФРАКТАЛЬНЫМИ ГРАФАМИ ${ }^{1}$

Павлов Дмитрий Алексеевич

к.ф.-м.н., доцент

РИНЦ SPIN-код, 8822-5089

dp.logic@gmail.com

Настоящая работа посвящена новому методу проектирования крупномасштабных структур транспортных сетей. Модель крупномасштабной транспортной сети строится на предфрактальных графах. В основе модели крупномасштабной транспортной сети лежит принцип иерархической организации территорий. Предфрактальный граф представляет собой некоторый конечный аналог фрактального графа, совмещающий свойства фрактала и графа. Некоторые задачи дискретной оптимизации на предфрактальных графах становятся полиномиально разрешимыми при определенных условиях. Снижение трудоемкости экстремальных задач на предфрактальных графах обусловленно тем, что на этих графах для некоторых задач наряду со свойством самоподобия появляется свойство наследственности. Используя это свойство, для задач на предфрактальных графах можно строить параллельные алгоритмы, трудоемкость которых на порядки ниже, чем у известных последовательных алгоритмов

КлючевЫе слова: ПРЕДФРАКТАЛЬНЫЙ ГРАФ, КРУПНОМАСШТАБНАЯ ТРАНСПОРТНАЯ СЕТЬ, ДИСКРЕТНАЯ ОПТИМИЗАЦИЯ
UDC 656.025: 519.17

Economics

\section{SIMULATION OF THE LARGE-SCALE TRANSPORT NETWORK BY PREFRACTAL GRAPHS}

Pavlov Dmitriy Alexeevich

Cand.Phys.-Math.Sci., associate professor

SPIN-code, 8822-5089

dp.logic@gmail.com

This work is devoted to a new method for designing large-scale structures of transport networks. The model of a large-scale transport network is built on prefractal graphs. The model of a large-scale transport network is based on the principle of hierarchical organization of territories. A prefractal graph is a finite analogue of a fractal graph combining the properties of a fractal and a graph. Some problems of discrete optimization on prefractal graphs become polynomially solvable under certain conditions. Reducing the complexity of extreme problems on prefractal graphs is due to the fact that on these graphs for some problems, along with the selfsimilarity property, the property of heredity appears. Using this property, it is possible to construct parallel algorithms for problems on prefractal graphs, the complexity of which is orders of magnitude lower than for known successive algorithms

Keywords: PREFRACTAL GRAPH, LARGESCALE TRANSPORT NETWORK, DISCRETE OPTIMIZATION

Doi: 10.21515/1990-4665-131-085

Транспорт - важный стратегический комплекс, в значительной степени определяющий мощь экономики страны и обеспечивающий нужды общества в перемещении людей и грузов. Эффективность транспорта и качество транспортного обслуживания [1] в значительной степени определяется организацией системы транспортных маршрутов. Чем шире география распределения и чем больше количество потребителей, тем сложнее организовать единый технологический процесс

\footnotetext{
${ }^{1}$ Исследование выполнено при финансовой поддержке РФФИ в рамках проекта 17-06-00282 a.
} 
грузовых или пассажирских перевозок. В силу специфики основных технологических процессов на транспорте, представляется перспективным использование математического аппарата теории графов. Однако при моделировании таких задач в «крупномасштабных» системах возникает ряд трудностей, к основным из которых относятся следующие:

1. нахождения системы оптимальных транспортных маршрутов при заданных критериях решается неэффективно для больших наборов данных, в смысле вычислительной сложности [2], с помощью методов дискретной оптимизации;

2. построенные математические модели должны учитывать структурную динамику [3] (в плане роста) происходящую с течением времени внутри структуры транспортной сети.

Для решения этих проблем предлагается использовать особый вид масштабно-инвариантных графов, называемых предфрактальными графами [4], которые дают существенный толчок для нового этапа развития и применения идей теории графов [5].

Интересные и оригинальные результаты были получены при моделировании сложных и иерархических систем самоподобными или фрактальными графами [4], которые представляют синтез идей синергетики [6] и нелинейной динамики [7], фракталов [8] и теории графов.

В работе исследуется пространственная структура крупномасштабных транспортных систем, которая определяется транспортными сетями. Транспортной сетью называется совокупность транспортных связей, по которым осуществляются пассажирские и грузовые перевозки.

Под понятием «крупномасштабных» систем [9] будем понимать класс сложных (больших) систем, характеризующийся комплексным межрегиональным взаимодействием элементов, распределенных на 
значительной территории, требующих для развития существенных затрат ресурсов и времени.

Наиболее естественным способом представления модели транспортной сети является граф, который сильно формализует исходную задачу, т. е. в полученной модели мы оперируем лишь терминами «вершина» и «ребро». Граф - это структура, состоящая из множества вершин и соединяющих их ребер. Множество вершин графа обозначаются точками на сети, наиболее важными для определения расстояний или маршрутов движения. Ребра графа - это отрезки транспортной сети, характеризующие наличие дорожной связи между соседними вершинами. Ребра графа характеризуются числами, называемыми весами ребер. Веса ребер могут иметь различный физический смысл, чаще всего это расстояние, издержки, стоимость проезда, но может использоваться, например, и время движения.

Граф, моделирующий транспортную сеть, обязательно должен быть связанным, чтобы всегда был путь из любой вершины в любую другую вершину.

В работе предлагается использовать в качестве модели пространственной структуры крупномасштабной транспортной сети предфрактальные графы в основе которых лежит, как выше было сказано, принцип масштабной инвариантности.

\section{Предфрактальный и фрактальный граф}

Определим понятие предфрактального графа индуктивно.

Обозначим через $H=(W, Q)$ - конечный связный $n$-вершинный граф с множеством вершин $W$ и множеством ребер $Q$, который назовем затравкой.

Под операцией «замещение вершины затравкой» (3В3) будем понимать обобщенную процедуру «расщепления вершины графа» [5]. 
Определим поэтапный процесс выполнения операции ЗВ3. На этапе $l=1$ графу $G_{1}=\left(V_{1}, E_{1}\right)$ соответствует затравка $H=(W, Q)$. Далее, на каждом следующем этапе $l=1,2, \ldots, s, \ldots, L$ к каждой вершине полученного на предыдущем шаге графа $G_{s}=\left(V_{s}, E_{s}\right)$ вновь применяется операция 3В3 в результате получается граф $G_{s+1}=\left(V_{s+1}, E_{s+1}\right)$. На этапе $l=L$ получим граф $G_{L}=\left(V_{L}, E_{L}\right)$, который назовем предфрактальным $(n, L)$-графом.

Процесс порождения предфрактального графа $G_{L}$ является процессом построения последовательности предфрактальных графов $G_{1}, G_{2}, \ldots, G_{L}$, называемой траекторией.

На рисунке 1 представлена траектория построения предфрактального граф $G_{3}=\left(V_{3}, E_{3}\right)$, порожденного 5-вершинной затравкой $H=(W, Q)$.

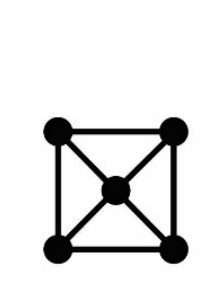

a) $G_{1}=H$

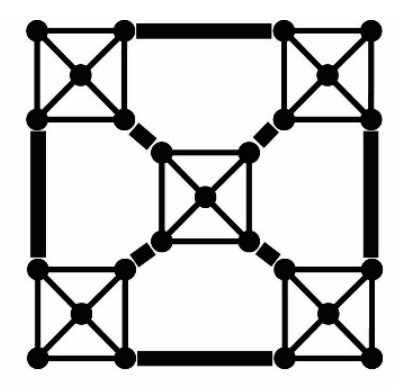

б) $G_{2}$

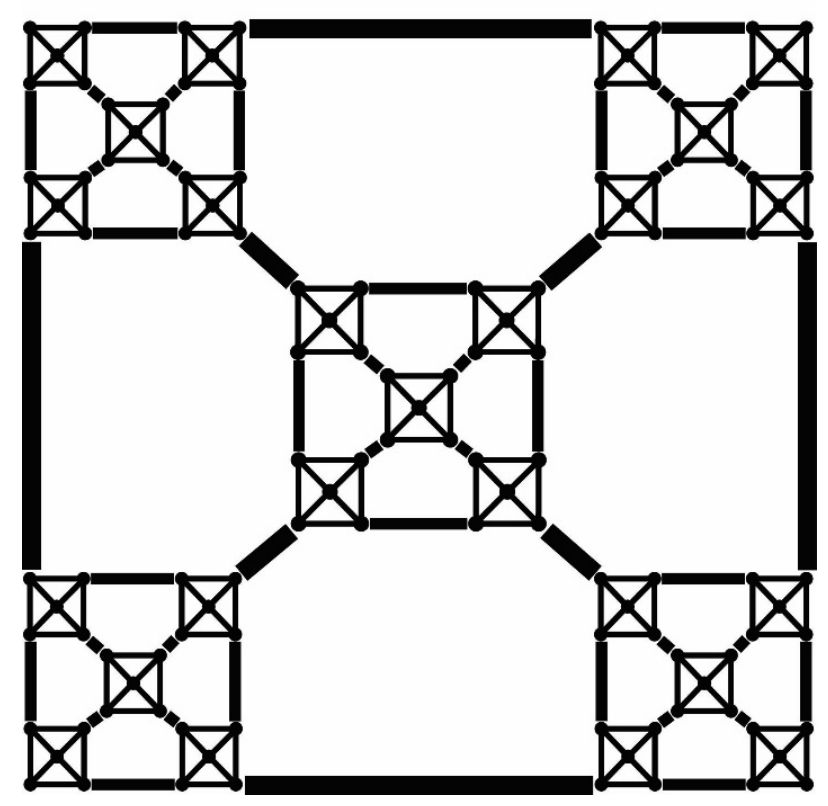

в) $G_{3}$

Рисунок 1 - Траектория предфрактального графа $G_{3}$ 
В процессе построения предфрактального графа смысл операции 3В3 состоит в том, что каждая расщепляемая вершина $v\left(V_{\perp} s\right.$ замещается затравкой. В результате, все ребра $e \in E_{\text {s }}$ сохраняются и называются старыми ребрами по отношению к ребрам $E_{s+1}$ появившимся при построении графа на этапе $l=s+\mathbf{1}$. При операции ЗВЗ все старые ребра инцидентные замещаемой вершине $v\left(V_{\downarrow} s\right.$ становятся регулярным или случайным образом инцидентными некоторым вершинам затравки, которая заместила вершину $v$.

Обобщением процесса порождения предфрактального графа является случай, когда вместо одной затравки Н для замещения выбираются затравки из множества $\mathrm{H}=\left\{H_{\downarrow} \mathbf{1}, H_{\downarrow} 2, \ldots, H_{\downarrow} T\right\}, T \geq 2$.

Ранг $L$ предфрактального графа $G_{L}=\left(V_{L}, E_{L}\right)$ определяет число этапов порождения графа.

$$
G=(V, E)
$$

Фрактальный граф определяется бесконечной траекторией.w

Следует отметить, что в качестве затравки может выбираться не только обыкновенный граф, но и мультиграф [5].

\section{Модель крупномасштабной структуры транспортной сети}

В основе модели крупномасштабной транспортной сети лежит принцип иерархической организации территорий (в нисходящем направлении).

Рассмотрим карту сети дорог в определенном порядке, начиная с более мелкого масштаба (в масштабе континента или страны), заканчивая более крупным масштабом города или населенного пункта. При исследовании крупномасштабной транспортной сети в масштабах страны 
на первом этапе рассмотрим дороги связывающие округа. В качестве автомобильных дорог на этом этапе выступают магистрали и федеральные трассы. На втором этапе, в масштабе округов, рассмотрим сеть дорог соединяющих субъекты округов (области, республики, края). Далее, на третьем этапе при уменьшении масштаба последовательно рассматриваются дороги связывающие определенные районы выбранного округа. Аналогично, при рассмотрении транспортной сети в масштабе района нас интересуют только дороги соединяющие населенные пункты этого района. На последнем этапе рассматриваются дороги в масштабе населенных пунктов.

Процесс рассмотрения структуры транспортной сети в указанном порядке напоминает траекторию построения предфрактального графа, порожденного множеством затравок $\mathrm{H}=\left\{H_{\downarrow} 1, H_{\downarrow} 2, \ldots, H_{\downarrow} T\right\}$.

При исследовании транспортной сети в масштабах страны в качестве затравки выбирается граф или в общем случае мультиграф вершинам которого соответствуют наиболее крупные территориальные единицы (регионы, федеральные округа). В качестве ребер используются автомобильные дороги соединяющие выбранные территориальные единицы.

Рассмотрим процесс построения предфрактального графа на примере транспортной сети дорог России. На рисунке 2 представлен мультиграф $G_{1}=\left(V_{1}, E_{1}\right) \quad$ - соответствующий структуре транспортной сети связывающий федеральные округа. 


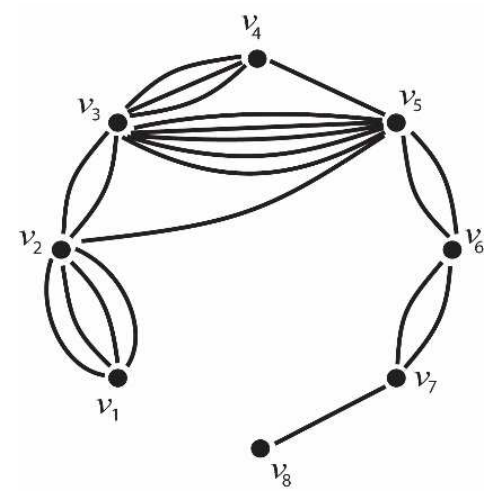

$v_{1}$ - Северо-Кавказский федеральный округ

$v_{2}-$ Южный федеральный округ

$v_{3}$ - Центральный федеральный округ

$v_{4}$ - Северо-Западный федеральный округ

$v_{5}-$ Приволжский федеральный округ

$v_{6}-$ Уральский федеральный округ

$v_{7}-$ Сибирский федеральный округ

$v_{8}-$ Дальневосточный федеральный округ

Рисунок 2 - Мультиграф структуры дорог федеральных округов

На рисунке 3 изображена структура предфрактального графа $G_{2}=\left(V_{2}, E_{2}\right)$ ранга $L=2$ порожденного на основе затравки $G_{1}=\left(V_{1}, E_{1}\right)$, в которой каждая вершина из множества $V_{1}=\left\{v_{1}, v_{2}, v_{3}, v_{4}, v_{5}, v_{6}, v_{7}, v_{8}\right\}$ замешается затравкой из множества $\mathrm{H}$, соответствующей структуре дорог транспортной сети внутри федерального округа соединяющих районы (края, области, республики). Жирными линиями на рисунке 3 изображены ребра ранга $L=1$, остальные ребра относятся к рангу $L=2$.

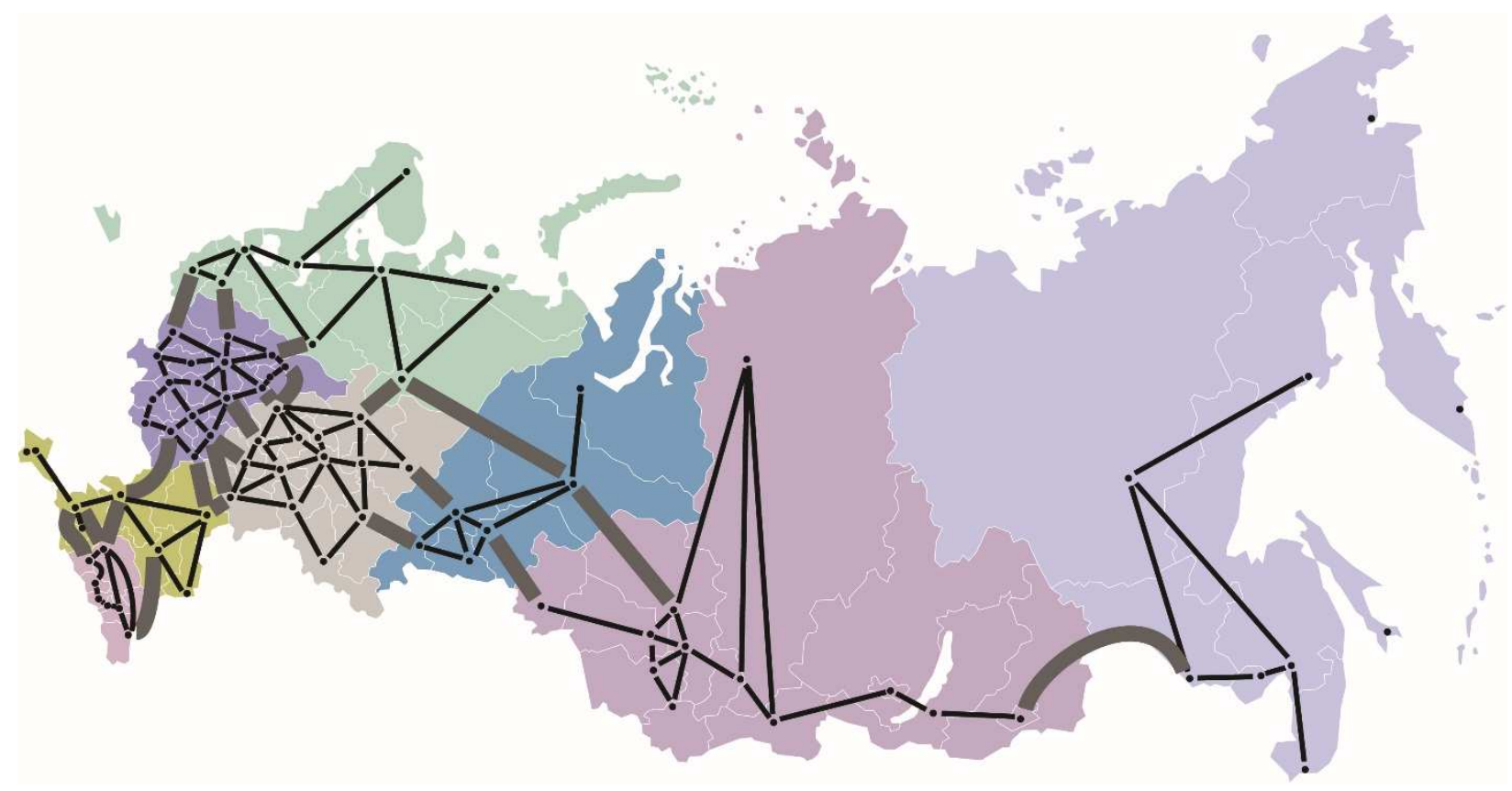

Рисунок 3 - Предфрактальный граф $G_{2}$ 
Следует отметить, что при рассмотрении иерархии вложенности карты дорог до уровня ранга $L=3$, затравки 2-го ранга, соответствующие территориальным единицам внутри федерального округа следует рассматривать как мультиграфы, где как правило существует не одна, а несколько дорог, связывающих соседствующие территориальные единицы.

\section{О вычислительной сложности алгоритмов, реализованных на предфрактальных графах}

Моделирование транспортной сети большой размерности с помощью предфрактальных графов позволяет строить эффективные алгоритмы благодаря свойству самоподобия.

В работах [10-14] построены алгоритмы вычислительная сложность которых на канонических предфрактальных графах в разы меньше подобных алгоритмов на графах. В работе [10] представлен алгоритм выделения остовного дерева минимального веса на предфрактальном графе $G_{L}$. Сравнив вычислительную сложность построенного алгоритма и алгоритма Прима [5] получим оценку $O\left(N^{2}\right)<O\left(N n^{2}\right)$, где $N=n^{L}$ множество вершин предфрактального $(n, L)$-графа ранга $L$, а $n$ - множество вершин затравки. Т. е. вычислительная сложность алгоритма выделения остовного дерева минимального веса на предфрактальном графе меньше вычислительной сложности алгоритма Прима в $n^{L-2}$ раз.

Здесь уместно отметить, что на пример, известная задача нахождения гамильтонова цикла становится полиномиально разрешимой на канонических предфрактальных графах с полной затравкой в случае, когда старые ребра не пересекаются. Снижение трудоемкости экстремальных задач на предфрактальных графах обусловлено тем, что на этих графах для некоторых задач наряду со свойством самоподобия появляется свойство наследственности. 
Используя это свойство, для задач на предфрактальных графах можно строить параллельные алгоритмы [16,17], трудоемкость которых на порядки ниже, чем у известных последовательных алгоритмов.

Отметим, что решение задач на предфрактальных графах, независимо от того представлен ли алгоритм как последовательный (использование одного процессора) или параллельный (использовании нескольких процессоров), вычислительная сложность этого алгоритма ниже чем у известных последовательных алгоритмов.

\section{Выводы}

В качестве модели карты дорог предлагается использовать предфрактальные графы, которые естественным образом отражают структуру связей при рассмотрении транспортной сети в различных масштабах (страны, регионов, областей). В задачах организации и планирования экономически эффективных грузовых и пассажирских перевозок использование предфрактальных графов позволяет решить ряд важных проблем:

1) существенно снизить трудоемкости некоторых известных алгоритмов дискретной оптимизации;

2) реализовывать параллельные алгоритмы с заметным снижением вычислительной сложности;

3) позволяют описать структурную динамику изучаемой системы в дискретном времени при росте структуры.

Таким образом, обосновано преимущество использование предфрактальных графов перед «классическими» графами в моделировании структуры крупномасштабных транспортных сетей. 


\section{Литература}

1. Горев А. Э. Основы теории транспортных систем: учеб. пособие / А. Э. Гореев; СПбГАСУ. - СПб., 2010. - 214 с.

2. М. Гэри. Вычислительные машины и труднорешаемые задачи / Гэри М., Джонсон Д. - М.: Мир, 1982.

3. Кочкаров А. М. Структурная динамика и исследование структурновременных характеристик дискретных систем / А. М. Кочкаров, А. А. Кочкаров, С. П. Никищенко // Известия ТРТУ. Тематический выпуск "Перспективные системы и задачи управления”. - Таганрог: ТРТУ, 2006. - № 3. - С. 235-238.

4. Кочкаров А. М. Распознавание фрактальных графов. Алгоритмический подход / А.М. Кочкаров. - Нижний Архыз: Изд. центр «CYGNUS», 1998. - 170 с.

5. Лекции по теории графов / В.А. Емеличев, О.И. Мельников, В.И. Сарванов, Р.И. Тышкевич. - М. : Наука, 1990. - 383 с.

6. Малинецкий Г. Г. Математические основы синергетики. Хаос, структуры, вычислительный эксперимент. М.: КомКнига, 2005.

7. Малинецкий Г.Г. Нелинейная динамика и проблемы прогноза / Г.Г. Малинецкий, С.П. Курдюмов // Вестник РАН. 2001.Т. 71, №3. - С. 210-224.

8. Кроновер Р. М. Фракталы и хаос в динамических системах. Основы теории / Р. М. Кроновер. - М., 2000. - 352 с.

9. Цвиркун А.Д. Управление развитием крупномасштабных систем в новых условиях // Проблемы управления. - 2003. - №1 - С. 34-43

10. Павлов Д.А., Кочкаров А.А. Об одной многокритериальной задачи покрытия минимального веса предфрактального графа простыми пересекающимися цепями. Препринт №200. РАН САО . Нижний Архыз. 2004.-12с.

11. Павлов Д.А., Кочкаров А.А. Узденов А.А. Об одной многокритериальной задаче выделения наибольших максимальных цепей на предфрактальных графах. Препринт №198. РАН САО. Нижний Архыз. 2004.-27с.

12. Павлов Д.А., Кочкаров Р.А. Алгоритм с оценками построения покрытий непересекающимися простыми цепями на предфрактальном графе. Препринт №199. РАН САО. Нижний Архыз. 2004.-24с.

13. Павлов Д.А., Салпагаров С.И. Многокритериальная задача выделения маршрутов на предфрактальном графе// Известия ТРГУ. - Таганрог: ТРГУ, 2004.

14. Павлов Д.А. Особенности многокритериальной оптимизации на предфрактальных графах: задача покрытия простыми цепями : монография / Д. А. Павлов. - Краснодар : КубГАУ, 2016. - 122 с.

15. Емеличев В. А. Лекции по теории графов / В. А. Емеличев и др.- М, Наука, 1990 - 384 с.

16. Кочкаров, А. А. Параллельные алгоритмы на предфрактальных графах: препринт / А. А. Кочкаров, Р. А. Кочкаров. - М. : Институт прикладной математики им. М.В. Келдыша РАН. - 2003. - № 84.

17. Кочкаров А. А., Кочкаров Р. А. Параллельный алгоритм поиска кратчайшего пути на предфрактальном графе / А. А. Кочкаров, Р. А. Кочкаров // Журн. вычислит. матем. и матем. физики. 2004. Т. 44. № 6. С. 1157-1162. 


\section{References}

1. Gorev A. Je. Osnovy teorii transportnyh sistem: ucheb. posobie / A. Je. Goreev; SPbGASU. - SPb., 2010. - 214 s.

2. M. Gjeri. Vychislitel'nye mashiny i trudnoreshaemye zadachi / Gjeri M., Dzhonson D. - M.: Mir, 1982.

3. Kochkarov A. M. Strukturnaja dinamika i issledovanie strukturno-vremennyh harakteristik diskretnyh sistem / A. M. Kochkarov, A. A. Kochkarov, S. P. Nikishhenko // Izvestija TRTU. Tematicheskij vypusk "Perspektivnye sistemy i zadachi upravlenija". Taganrog: TRTU, 2006. - № 3. - S. 235-238.

4. Kochkarov A. M. Raspoznavanie fraktal'nyh grafov. Algoritmicheskij podhod / A.M. Kochkarov. - Nizhnij Arhyz: Izd. centr «CYGNUS», 1998. - 170 c.

5. Lekcii po teorii grafov / V.A. Emelichev, O.I. Mel'nikov, V.I. Sarvanov, R.I. Tyshkevich. - M. : Nauka, 1990. - 383 s.

6. Malineckij G. G. Matematicheskie osnovy sinergetiki. Haos, struktury, vychislitel'nyj jeksperiment. M.: KomKniga, 2005.

7. Malineckij G.G. Nelinejnaja dinamika i problemy prognoza / G.G. Malineckij, S.P. Kurdjumov // Vestnik RAN. 2001.T. 71, №3. - S. 210-224.

8. Kronover P. M. Fraktaly i haos v dinamicheskih sistemah. Osnovy teorii / P. M. Kronover. - M., 2000. - 352 s.

9. Cvirkun A.D. Upravlenie razvitiem krupnomasshtabnyh sistem $\mathrm{v}$ novyh uslovijah // Problemy upravlenija. - 2003. - №1. - S. 34-43

10. Pavlov D.A., Kochkarov A.A. Ob odnoj mnogokriterial'noj zadachi pokrytija minimal'nogo vesa predfraktal'nogo grafa prostymi peresekajushhimisja cepjami. Preprint №200. RAN SAO . Nizhnij Arhyz. 2004.-12s.

11. Pavlov D.A., Kochkarov A.A. Uzdenov A.A. Ob odnoj mnogokriterial'noj zadache vydelenija naibol'shih maksimal'nyh cepej na predfraktal'nyh grafah. Preprint №198. RAN SAO. Nizhnij Arhyz. 2004.-27s.

12. Pavlov D.A., Kochkarov R.A. Algoritm s ocenkami postroenija pokrytij neperesekajushhimisja prostymi cepjami na predfraktal'nom grafe. Preprint №199. RAN SAO. Nizhnij Arhyz. 2004.-24s.

13. Pavlov D.A., Salpagarov S.I. Mnogokriterial'naja zadacha vydelenija marshrutov na predfraktal'nom grafe// Izvestija TRGU. - Taganrog: TRGU, 2004.

14. Pavlov D.A. Osobennosti mnogokriterial'noj optimizacii na predfraktal'nyh grafah: zadacha pokrytija prostymi cepjami : monografija / D. A. Pavlov. - Krasnodar : KubGAU, 2016. - 122 s.

15. Emelichev V. A. Lekcii po teorii grafov / V. A. Emelichev i dr.- M, Nauka, $1990-384 \mathrm{~s}$.

16. Kochkarov, A. A. Parallel'nye algoritmy na predfraktal'nyh grafah: preprint / A. A. Kochkarov, R. A. Kochkarov. - M. : Institut prikladnoj matematiki im. M.V. Keldysha RAN. - 2003. - № 84 .

17. Kochkarov A. A., Kochkarov R. A. Parallel'nyj algoritm poiska kratchajshego puti na predfraktal'nom grafe / A. A. Kochkarov, R. A. Kochkarov // Zhurn. vychislit. matem. i matem. fiziki. 2004. T. 44. № 6. S. 1157-1162. 